\title{
Multi-Bernoulli Filtering for Initially Unresolved Targets in Clutter
}

\author{
Qin Lu ${ }^{\mathrm{a}}$, Karl Granström ${ }^{\mathrm{b}}$, Yaakov Bar-Shalom ${ }^{\mathrm{a}}$, and Peter Willett ${ }^{\mathrm{a}}$ \\ ${ }^{a}$ Department of Electrical and Computer Engineering, University of Connecticut, USA \\ ${ }^{\mathrm{b}}$ Chalmers University of Technology, Sweden
}

\begin{abstract}
Multiple target tracking (MTT) is a challenging task that aims to estimate the number of targets and their states in the presence of process noise, measurement noise and data association uncertainty. This paper considers a special MTT problem characterized by additional complexity. In this problem, multiple targets are launched simultaneously in nearby locations at the same speed with slightly different directions. As the distances between the initial locations of these targets are smaller than the resolution of the sensor, this results in merged measurements, i.e., unresolved tracks at the very beginning. To deal with this problem, the recently proposed Multi-Bernoulli (MB) filter is applied. Using a model for the merged measurements, simulation results with 2-D Cartesian measurements in an optical sensor's focal plane in the presence of clutter show that the initially unresolved tracks become resolved with MB filtering a few time steps after the measurements become resolved. Thus, the MB filter is capable of keeping track of the number of targets and their corresponding states when they are initially unresolved.
\end{abstract}

Keywords: Multiple Target Tracking, Unresolved Targets, Multi-Bernoulli Filtering, Clutter

\section{INTRODUCTION}

Multiple target tracking (MTT) is the processing of sets of measurements obtained from one or several sensors in order to maintain estimates of targets' current states. The task is complicated by the fact that - in addition to noise, missed detections and clutter - the number of targets is unknown and possibly time-varying.

Broadly speaking there are three different approaches to multiple target tracking: Multiple Hypothesis Tracking (MHT) [4], Joint Probabilistic Data Association (JPDA) [1], and Random Finite Sets (RFS) [11,12]. The MHT type approaches involve propagating target track hypotheses in time and calculating their likelihoods, the JPDA type approaches blend data association probabilities on a scan-by-scan basis, and the RFS type approaches rely on modeling the targets and the measurements as random sets.

The RFS approach is the latest development for MTT, the basic idea of which is to treat each multi-target state as an RFS. The Bayes multiobject filter (BMF) is the original RFS-type filter that propagates and updates the density (pdf) of the multiobject state in time. Because of the computational complexity of the data association problem, the BMF is generally considered infeasible to implement. Computationally feasible approximations are the Probability Hypothesis Density (PHD) filters [13], the Cardinalized PHD (CPHD) filters [14], and the multi-Bernoulli (MB) filters [17, 18, 21-23,25].

The PHD filters recursively estimate the first order moment of the multiobject state, called the PHD intensity, under an assumed Poisson distribution for the cardinality. The CPHD filters recursively estimate the PHD and also a cardinality distribution that can be non-Poisson. A known drawback of the PHD filter is the high variance of its cardinality estimate, as a consequence of the underlying Poisson assumption. The CPHD filters are known to have better cardinality estimates, but become susceptible to a "spooky effect" [6,12], a phenomenon manifested by PHD mass shifted from undetected targets to detected targets that are far enough away that they ought to be statistically insulated. The MB filters $[17,18,21,23,25]$ propagate the parameters of a multi-Bernoulli

This work was supported by ARO Grant W911-NF-10-1-0369.

Signal Processing, Sensor/Information Fusion, and Target Recognition XXV, edited by Ivan Kadar, Proc. of SPIE Vol. 9842, 98421J · C 2016 SPIE · CCC code: 0277-786X/16/\$18 · doi: 10.1117/12.2229638 
distribution that approximate the posterior multi-target distribution. The MB filters estimate, for each target, the location and probability of existence; and they are known to be capable of matching the CPHD filters' cardinality performance without being susceptible to the "spooky effect".

Ultimately the desired output from an MTT algorithm is a set of estimated trajectories (tracks), where a trajectory is defined as the sequence of states from the time the target appears to the time it disappears. Both the MHT and JPDA type algorithms estimate trajectories. In their most basic forms, none of the PHD [13], CPHD [14] nor multi-Bernoulli filters [22] formally estimates target trajectories — only a set of target state estimates is supplied at each time step - however, target trajectories can be obtained, e.g. using labeling schemes [16]. With the introduction of labeled RFSs it has become possible to obtain trajectory estimates without the need for post-processing, leading to the Generalized Labeled Multi-Bernoulli (GLMB) filter [21], and its computationally efficient approximation the Labeled Multi-Bernoulli (LMB) filter [18]. An analysis of the approximation error in the GLMB filter is given in [24].

This paper considers the application of the MB filter to a MTT problem with additional complexity. A scenario of closely spaced objects, launched simultaneously with slight difference in the velocity vector, is considered. As the distances between the initial locations of the targets are smaller than the resolution of the sensor, this results in merged measurements. As the scenario proceeds, the targets become well separated with resolved measurements. We start by considering a simplified version using nearly constant velocity models with 2D Cartesian measurements in the focal plane of an optical sensor. Simulation results are provided to validate the capability of MB filter to track both the number and states of the targets when the initially unresolved target-originated measurements become resolved. A similar problem of group to object tracking (GTO) was explored in [9] by means of MHT, where GTO tracking is the process of tracking clusters of unresolved or nearly resolved objects as a group and tracking each object separately when they become clearly resolved.

The rest of the paper is organized as follows. The models of target dynamics and merged measurements along with the propagation equations for the Bayes multi-object filter are introduced in Section 2. The detailed implementation of the MB filter is provided in Section 3 . Simulation results are provided in Section 4 to validate the capability of MB filter to the MTT problem with merged measurements. Concluding remarks are made in Section 5 to summarize the paper.

\section{SYSTEM MODEL}

\subsection{Dynamic Model}

Let $\mathbf{x}_{k}^{i}$ denote the state of the $i$ th target at time step $k$. The nearly constant velocity model, or white noise acceleration (WNA) model in [1], in two-dimensional Cartesian coordinates in an optical sensor's focal plane is considered in this paper. Therefore, $\mathbf{x}_{k}^{i}=\left[\xi_{k}^{i}, \eta_{k}^{i}, \dot{\xi}_{k}^{i}, \dot{\eta}_{k}^{i}\right]^{\prime}$, where $\left[\xi_{k}^{i}, \eta_{k}^{i}\right]$ is the position of the $i$ th target at time step $k$ in Cartesian coordinates, and $\left[\dot{\xi}_{k}^{i}, \dot{\eta}_{k}^{i}\right]$ is the corresponding velocity. Also, all the targets have the following dynamic model as in [1]

$$
\mathbf{x}_{k+1}^{i}=\mathbf{F} \mathbf{x}_{k}^{i}+v_{k}^{i}
$$

where $\mathbf{F}$ is the state transition matrix represented by the sampling time $T$ in $(2)$ and $v_{k}^{i}$ is the process noise, which is assumed Gaussian distributed with zero mean and covariance $\mathbf{Q}$, characterized by $T$ and acceleration noise standard deviation $\sigma_{v}$ in (3).

$$
\begin{gathered}
\mathbf{F}=\left[\begin{array}{cccc}
1 & 0 & T & 0 \\
0 & 1 & 0 & T \\
0 & 0 & 1 & 0 \\
0 & 0 & 0 & 1
\end{array}\right] \\
\mathbf{Q}=\left[\begin{array}{cccc}
\frac{T^{4}}{4} & 0 & \frac{T^{3}}{2} & 0 \\
0 & \frac{T^{4}}{4} & 0 & \frac{T^{3}}{2} \\
\frac{T^{3}}{2} & 0 & T^{2} & 0 \\
0 & \frac{T^{3}}{2} & 0 & T^{2}
\end{array}\right] \sigma_{v}^{2}
\end{gathered}
$$


Suppose the number of targets at time $k$ is $N_{k}^{x}$, the target state set can be denoted as

$$
\mathbf{X}_{k}=\left\{\mathbf{x}_{k}^{i}\right\}_{i=1}^{N_{k}^{x}}
$$

Therefore, the number of targets at time step $k$ is equivalent to the cardinality of set $\left|\mathbf{X}_{k}\right|$, that is, $N_{k}^{x}=\left|\mathbf{X}_{k}\right|$. Due to the birth and death of targets, $N_{k}^{x}$ is a time-varying discrete random process. For MTT, the goal is to estimate $N_{k}^{x}$ and $\mathbf{X}_{k}$ jointly at each time step.

\subsection{Measurement Model}

For each target, the true Cartesian position is

$$
\mathbf{y}_{k}^{i}=\mathbf{H} \mathbf{x}_{k}^{i}
$$

where

$$
\mathbf{H}=\left[\begin{array}{llll}
1 & 0 & 0 & 0 \\
0 & 1 & 0 & 0
\end{array}\right]
$$

is the observation matrix.

We consider a scenario of closely spaced objects launched simultaneously at the same speed with slightly different directions. For the first few time steps, the distances of the true positions of some of the targets are too small to be resolved by the sensor so that only one merged measurement is retained. The threshold of resolution for each coordinate is set to $3 \sigma_{r}$, where $\sigma_{r}$ is the common measurement noise standard deviation for each resolved target. Two targets are considered unresolved ${ }^{*}$ if the distances in all the coordinates between them are less than $3 \sigma_{r}$.

Based on the distances in target separation, the targets are divided into different groups. Let $M_{k}^{j}, j=$ $1, \ldots, N_{k}^{m}$, be the $j$ th set containing the indices of the merged targets whose true positions at time $k$ cannot be resolved. Target $p$ belongs to set $M_{k}^{j}$ if there exists another target $q$ whose distance from target $p$ in every coordinate is smaller than $3 \sigma_{r}$. For targets from different sets $M_{k}^{j}$, the distance in at least one coordinate should be larger than $3 \sigma_{r}$. For all the targets within set $M_{k}^{j}$, there is only one merged measurement, whose mean is taken as the average of true positions of all the targets within and the associated measurement noise covariance is $\mathbf{R}=\sigma_{r}^{2} \mathbf{I}_{2}$ times the cardinality of the set $M_{k}^{j}$, where $\mathbf{I}_{2}$ is the $2 \times 2$ identity matrix. That is

$$
\mathbf{y}_{k}^{M, j}=\overline{\mathbf{y}}_{k}^{j}+w_{k}^{M, j}
$$

where $\overline{\mathbf{y}}_{k}^{j}$ is the average position for all targets within set $M_{k}^{j}$ and $w_{k}^{M, j}$ is the measurement noise whose covariance is $\left|M_{k}^{j}\right| \mathbf{R}$. Note that the superscript " $M$ " in $\mathbf{y}_{k}^{M, j}$ and $w_{k}^{M, j}$ represents "Merged". When there is only one target in set $M_{k}^{j}$, namely, $\left|M_{k}^{j}\right|=1$, this is equivalent to the measurement model for a single resolved target and the target is resolved from all of the remaining targets. Consequently, the number of resolved targets is the number of sets $\left(M_{k}^{j} \mathrm{~s}\right)$ with only one element. The set of all the target originated measurements at time $k$ can be then denoted as

$$
\mathbf{Y}_{k} \triangleq\left\{\mathbf{y}_{k}^{M, j}\right\}_{j=1}^{N_{k}^{m}}
$$

In addition to the target originated measurements, there also exist clutter measurements $\mathbf{C}_{k}$. And the measurement set is the union of $\mathbf{C}_{k}$ and $\mathbf{Y}_{k}$ as

$$
\mathbf{Z}_{k}=\mathbf{C}_{k} \cup \mathbf{Y}_{k} \triangleq\left\{\mathbf{z}_{k}^{j}\right\}_{j=1}^{N_{k}^{z}}
$$

where $N_{k}^{z}=\left|\mathbf{Z}_{k}\right|$ is the cardinality of the measurement set at time $k$. Note that the measurement origin is assumed unknown and the sets above are without order. $\mathbf{Z}^{k}$ denotes all measurement sets up to time $k$ :

$$
\mathbf{Z}^{k}=\left\{\mathbf{Z}_{\kappa}\right\}_{\kappa=1}^{k}
$$

${ }^{*}$ Here, we define the merged measurement noise variance heuristically. In the future, we will develop a physics-based model for it. 


\subsection{Bayes Multi-Object Filter for MTT}

The Bayes multi-object filter is the centerpiece of RFS-type filter that propagates and updates the probability density function (pdf) of the multi-object state in time. Suppose the posterior multi-object distribution at time step $k-1$ is $f\left(\mathbf{X}_{k-1} \mid \mathbf{Z}^{k-1}\right)$, the predicted multi-object distribution at time $k$ is given by the Chapman-Kolmogorov equation as [7]

$$
f\left(\mathbf{X}_{k} \mid \mathbf{Z}^{k-1}\right)=\int f\left(\mathbf{X}_{k} \mid \mathbf{X}_{k-1}\right) f\left(\mathbf{X}_{k-1} \mid \mathbf{Z}^{k-1}\right) \delta \mathbf{X}_{k-1}
$$

where $f\left(\mathbf{X}_{k} \mid \mathbf{X}_{k-1}\right)$ is the multi-object transition density. Multi-object prediction involves modeling the time evolution of surviving targets (targets that remain in the surveillance area), target death (targets that do not remain), and target birth (new targets that appear in the surveillance area). The targets are assumed to evolve over time independently. The integral in (11) is a set integral, defined as [7]

$$
\int f(\mathbf{X}) \delta \mathbf{X}=\sum_{n=0}^{\infty} \frac{1}{n !} \int f\left(\left\{\mathbf{x}^{1}, \ldots, \mathbf{x}^{n}\right\}\right) \mathrm{d}\left(\mathbf{x}^{1}, \ldots, \mathbf{x}^{\mathrm{n}}\right)
$$

The posterior multi-object distribution at time $t_{k}$ is given by the Bayes update

$$
f\left(\mathbf{X}_{k} \mid \mathbf{Z}^{k}\right)=\frac{f\left(\mathbf{Z}_{k} \mid \mathbf{X}_{k}\right) f\left(\mathbf{X}_{k} \mid \mathbf{Z}^{k-1}\right)}{\int f\left(\mathbf{Z}_{k} \mid \mathbf{X}_{k}\right) f\left(\mathbf{X}_{k} \mid \mathbf{Z}^{k-1}\right) \delta \mathbf{X}_{k}}
$$

The multi-object measurement set density $f\left(\mathbf{Z}_{k} \mid \mathbf{X}_{k}\right)$ involves modeling target detection, measurement noise, and clutter measurements.

The cardinality of the clutter measurements set $N_{k}^{c}$ is typically modeled as a Poisson random variable with intensity $\lambda_{c}$, and each clutter measurement is assumed to be distributed with pdf $g_{c}(\mathbf{z})$. The clutter set pdf is then $[11]$

$$
\kappa\left(\mathbf{C}_{k}\right)=e^{-\lambda_{c}} \prod_{j=1}^{N_{k}^{c}} \lambda_{c} g_{c}\left(\mathbf{z}_{k}^{j}\right)
$$

This paper considers so called point targets, meaning that the $i$ th target measurement set $\mathbf{W}_{k}\left(\mathbf{x}_{k}^{i}\right)$ is a Bernoulli RFS that is empty $(=\emptyset)$ with probability $1-p_{\mathrm{D}}\left(\mathbf{x}_{k}^{i}\right)$, and with probability $p_{\mathrm{D}}\left(\mathbf{x}_{k}^{i}\right)$ the set contains a single measurement $\mathbf{z}_{k}$ originating from $\mathbf{x}_{k}^{i}$, distributed according to the pdf $g_{x}\left(\mathbf{z}_{k} \mid \mathbf{x}_{k}^{i}\right)$.

Under the assumption of Poisson clutter and independent point target measurements, the measurement set pdf is $[11]$

$$
\begin{aligned}
& f\left(\mathbf{Z}_{k} \mid \mathbf{X}_{k}\right)=e^{-\lambda_{c}}\left[\prod_{j=1}^{N_{k}^{z}} \lambda_{c} g_{c}\left(\mathbf{z}_{k}^{j}\right)\right]\left[\prod_{i=1}^{N_{k}^{x}}\left(1-p_{\mathrm{D}}\left(\mathbf{x}_{k}^{i}\right)\right)\right] \\
& \times \sum_{\theta \in \Theta} \prod_{i: \sigma_{i}>0} \frac{p_{\mathrm{D}}\left(\mathbf{x}_{k}^{i}\right)}{1-p_{\mathrm{D}}\left(\mathbf{x}_{k}^{i}\right)} \frac{g_{x}\left(\mathbf{z}_{k}^{\sigma_{i}} \mid \mathbf{x}_{k}^{i}\right)}{\lambda_{c} g_{c}\left(\mathbf{z}_{k}^{\sigma_{i}}\right)} \\
& =\sum_{\theta \in \Theta} e^{-\lambda_{c}}\left[\prod_{j: \nexists \sigma_{i}=j} \lambda_{c} g_{c}\left(\mathbf{z}_{k}^{j}\right)\right]\left[\prod_{i: \sigma_{i}=0}\left(1-p_{\mathrm{D}}\left(\mathbf{x}_{k}^{i}\right)\right)\right] \\
& \quad \times\left[\prod_{i: \sigma_{i}>0} p_{\mathrm{D}}\left(\mathbf{x}_{k}^{i}\right) g_{x}\left(\mathbf{z}_{k}^{\sigma_{i}} \mid \mathbf{x}_{k}^{i}\right)\right]
\end{aligned}
$$

Here $\theta=\left\{\sigma_{i}\right\}$, defined as in [11], is a set of associations $\sigma_{i}$, where $\sigma_{i}=0$ if target $\mathbf{x}_{k}^{i}$ is not associated to any measurement, and $\sigma_{i}=j$ if target $\mathbf{x}_{k}^{i}$ is associated to measurement $\mathbf{z}_{k}^{j}$. The set of all associations $\theta$ is denoted $\Theta$. 
Because of the computational complexity of the data association problem, it is generally considered infeasible to implement and use a multi-object filter without approximating the data association problem in some way. This paper considers a computationally feasible approximation, the multi-object particle multi-Bernoulli (MOP-MB) filter, which was proposed in [8] and will be discussed in details in the next section.

\section{MULTI-OBJECT PARTICLE MULTI-BERNOULLI FILTER}

In this section, we present the details of MOP-MB filter for MTT. The birth process is assumed unknown and an adaptive birth process is used $[18,19]$. We assume that the clutter is uniformly distributed in the surveillance area, namely, $g_{c}(\mathbf{z})=1 / V$, where $V$ is the volume of the surveillance region. The probability of detection $p_{\mathrm{D}}$ and probability of survival $p_{\mathrm{S}}$ are assumed the same for all the targets at all time steps.

\subsection{Initialization and Target Birth Prediction}

All the measurements obtained from the first step are regarded as potential targets. As a kinematic motion model and Cartesian measurements are assumed, the states of the birth targets are taken as each position measurement appended with zero velocity if there is no prior information about the velocity. Otherwise, if prior knowledge is available about the initial velocity, it can be used here instead. Similarly, for subsequent time steps, measurements that are not associated with any existing MB component are taken as potential new targets. The Gaussian MB density representing new targets (due to birth, or spawning) at time step $k-1$ is

$$
\left\{\left(w_{b}, m_{k-1}^{b, i}, P_{b}\right)\right\}_{i=1}^{N_{k-1}^{b}}
$$

where $m_{k-1}^{b, i}=\left[\begin{array}{lll}z_{k-1, b}^{\prime} & 0 & 0\end{array}\right]^{\prime}$ with $z_{k-1, b}$ being a measurement associated with no existing MB component, the weight $w_{b}$ and the covariance $P_{b}$ are user-defined parameters that are equal for all birth components. Note that this handles both new target birth and target spawning.

\subsection{Prediction}

Suppose the updated MB components at time $k-1$ are denoted as

$$
\left\{\left(w_{k-1 \mid k-1}^{i}, m_{k-1 \mid k-1}^{i}, P_{k-1 \mid k-1}^{i}\right)\right\}_{i=1}^{N_{k-1 \mid k-1}^{M B}}
$$

The predicted Gaussian MB density at time step $k$ is based on the union of the surviving targets MB density (17) and the new targets MB density (16). Merging the born targets set (16) with the surviving targets set (17), the number of predicted targets is

$$
N_{k \mid k-1}^{M B}=N_{k-1 \mid k-1}^{M B}+N_{k-1}^{b}
$$
is

Following the MB prediction used in the LMB filter [18], the predicted Gaussian MB density for each target

$$
\left\{\left(w_{k \mid k-1}^{i}, m_{k \mid k-1}^{i}, P_{k \mid k-1}^{i}\right)\right\}_{i=1}^{N_{k \mid k-1}^{M B}}
$$

where

$$
\begin{aligned}
w_{k \mid k-1}^{i} & =p_{\mathrm{S}} w_{k-1 \mid k-1}^{i} \\
m_{k \mid k-1}^{i} & =\mathbf{F} m_{k-1 \mid k-1}^{i} \\
P_{k \mid k-1}^{i} & =\mathbf{F} P_{k-1 \mid k-1}^{i} \mathbf{F}^{\prime}+\mathbf{Q}
\end{aligned}
$$




\subsection{Update}

The update is an adaptation of the PHD update in [10] and has three main steps:

1. The predicted MB density is used to create a particle approximation of the predicted multi-object density $f\left(\mathbf{X}_{k} \mid \mathbf{Z}^{k-1}\right)$.

2. Update each multi-object particle using the multi-object update (13), with an approximation for the data association computed using the auction algorithm [3].'

3. Use the posterior multi-object particles to approximate the posterior MB density.

To mitigate computational cost, measurement gating should be used to define groups of estimates and measurements, such that given the gating decisions the groups are statistically independent. The update is then performed separately for each group. The details of the update are below.

\subsubsection{Approximate predicted multi-object density}

Given the predicted Gaussian MB density in (19), we approximate the predicted multi-object density by $M$ multi-object particles $\mathbf{X}_{k \mid k-1}^{\ell}$. For the $\ell$ th multi-object particle, a set $I_{\ell}$ that indicates the existence of each MB component is obtained by random sampling. The existence of the $i$ th target is such that $i \in I_{\ell}$ if $u_{\ell}^{i} \leq w_{k \mid k-1}^{i}$, where $u_{\ell}^{i}$ is randomly sampled from the uniform distribution $\mathcal{U}(0,1)$. Consequently, the meaning of the set $I_{\ell}$ is that in the $\ell$ th particle the $i$ th predicted Gaussian component is included with probability $w_{k \mid k-1}^{i}$. For each particle, the cardinality of the targets is the cardinality of the set $I_{\ell}$. The predicted multi-object distribution in (11) is approximated by

$$
f\left(\mathbf{X}_{k} \mid \mathbf{Z}^{k-1}\right) \approx \sum_{\ell=1}^{M} \mathcal{W}_{k \mid k-1}^{\ell} \phi_{\mathbf{X}_{k}}\left(\mathbf{X}_{k \mid k-1}^{\ell}\right)
$$

where $\mathcal{W}_{k \mid k-1}^{\ell}$ is the prior weight of each MOP, which is assumed equal for all the particles, namely, $\mathcal{W}_{k \mid k-1}^{\ell}=$ $M^{-1}, \forall \ell$. For the $\ell$ th particle,

$$
\phi \mathbf{X}_{k}\left(\mathbf{X}_{k \mid k-1}^{\ell}\right)=\prod_{i \in I_{\ell}} \mathcal{N}\left(\mathbf{x}_{i} ; m_{k \mid k-1}^{i}, P_{k \mid k-1}^{i}\right) \delta\left[\left|\mathbf{X}_{k}\right|-\left|\mathbf{X}_{k \mid k-1}^{\ell}\right|\right]
$$

where $\delta[\cdot]$ is the Kronecker delta function. Therefore $\phi_{\mathbf{X}_{k}}\left(\mathbf{X}_{k \mid k-1}^{\ell}\right)=0$ if $\left|\mathbf{X}_{k}\right| \neq\left|\mathbf{X}_{k \mid k-1}^{\ell}\right|$ and

$$
\phi \mathbf{X}_{k}\left(\mathbf{X}_{k \mid k-1}^{\ell}\right)=\prod_{i \in I_{\ell}} \mathcal{N}\left(\mathbf{x}_{i} ; m_{k \mid k-1}^{i}, P_{k \mid k-1}^{i}\right)
$$

if $\left|\mathbf{X}_{k}\right|=\left|\mathbf{X}_{k \mid k-1}^{\ell}\right|$.

When sampling, one can get the same particle again, in which case computations should be saved by only considering the unique particles.

\subsubsection{Update}

Given the particle approximation of the predicted multi-object density (23), the posterior multi-object density given by the Bayes update (13) can be then propagated as

$$
f\left(\mathbf{X}_{k} \mid \mathbf{Z}^{k}\right)=\frac{\sum_{\ell=1}^{M} f\left(\mathbf{Z}_{k} \mid \mathbf{X}_{k}\right) \phi_{\mathbf{X}_{k}}\left(\mathbf{X}_{k \mid k-1}^{\ell}\right)}{\sum_{\ell=1}^{M} \int f\left(\mathbf{Z}_{k} \mid \mathbf{X}_{k}\right) \phi_{\mathbf{X}_{k}}\left(\mathbf{X}_{k \mid k-1}^{\ell}\right) \delta \mathbf{X}_{k}}
$$

\footnotetext{
${ }^{\dagger}$ JPDA association probabilities [2], cheap JPDA [5] or Murty's algorithm [15] can also be used.
} 
Using the measurement set pdf (15) and the definition of $\phi_{\mathbf{X}_{k}}\left(\mathbf{X}_{k \mid k-1}^{\ell}\right)$ in (24), for each multi-object particle we have

$$
f\left(\mathbf{Z}_{k} \mid \mathbf{X}_{k}\right) \phi_{\mathbf{X}_{k}}\left(\mathbf{X}_{k \mid k-1}^{\ell}\right)=0
$$

if $\left|\mathbf{X}_{k}\right| \neq\left|\mathbf{X}_{k \mid k-1}^{\ell}\right|$, and when $\left|\mathbf{X}_{k}\right|=\left|\mathbf{X}_{k \mid k-1}^{\ell}\right|$ we have

$$
\begin{aligned}
& f\left(\mathbf{Z}_{k} \mid \mathbf{X}_{k}\right) \phi_{\mathbf{X}_{k}}\left(\mathbf{X}_{k \mid k-1}^{\ell}\right) \\
= & \sum_{\theta \in \Theta} e^{-\lambda_{c}}\left[\prod_{j: \nexists \sigma_{i}=j} \frac{\lambda_{c}}{V}\right]\left[\prod_{i \in I_{\ell}: \sigma_{i}=0}\left(1-p_{\mathrm{D}}\right)\right] \\
& \times\left[\prod_{i \in I_{\ell}: \sigma_{i}>0} p_{\mathrm{D}} \mathcal{N}\left(\mathbf{z}_{k}^{\sigma_{i}} ; m_{k \mid k-1}^{i}, P_{k \mid k-1}^{i}\right)\right] \\
& \times\left[\prod_{i \in I_{\ell}} \mathcal{N}\left(\mathbf{x}_{k}^{i} ; m_{k \mid k-1}^{i}, P_{k \mid k-1}^{i}\right)\right] \\
= & \sum_{\theta \in \Theta} \mathcal{L}_{k \mid k-1}^{\ell}(\theta) \prod_{i \in I_{\ell}} \mathcal{N}\left(\mathbf{x}_{i} ; m_{k \mid k}^{i, \sigma_{i}}, P_{k \mid k}^{i, \sigma_{i}}\right) \\
= & \sum_{\theta \in \Theta} \mathcal{L}_{k \mid k-1}^{\ell}(\theta) \phi \mathbf{x}_{k}\left(\mathbf{X}_{k \mid k-1}^{\ell, \theta}\right)
\end{aligned}
$$

The likelihoods of the association events, given the MOPs, are

$$
\begin{aligned}
& \mathcal{L}_{k \mid k-1}^{\ell}(\theta)=e^{-\lambda_{c}}\left(\frac{\lambda_{c}}{V}\right)^{N_{F A}(\theta)}\left[\prod_{i \in I_{\ell}: \sigma_{i}=0}\left(1-p_{\mathrm{D}}\right)\right] \\
& \times\left[\prod_{i \in I_{\ell}: \sigma_{i}>0} p_{\mathrm{D}}\right]\left[\prod_{i \in I_{\ell}: \sigma_{i}>0} \mathcal{N}\left(\mathbf{z}_{k}^{\sigma_{i}} ; \hat{\mathbf{z}}_{k}^{i}, S_{k}^{i}\right)\right]
\end{aligned}
$$

where $N_{F A}(\theta)$ is the number of measurements that are not associated to a target, and the propagation equations of Kalman filter for each target or MB component are

$$
\begin{aligned}
S_{k}^{i} & =\mathbf{H} P_{k \mid k-1}^{i} \mathbf{H}^{\prime}+\mathbf{R} \\
K_{k}^{i} & =P_{k \mid k-1}^{i} \mathbf{H}^{\prime}\left(S_{k}^{i}\right)^{-1} \\
\hat{\mathbf{z}}_{k}^{i} & =\mathbf{H} m_{k \mid k-1}^{i} \\
m_{k \mid k}^{i, \sigma_{i}} & =m_{k \mid k-1}^{i}+K_{k}^{i}\left(\mathbf{z}_{k}^{\sigma_{i}}-\hat{\mathbf{z}}_{k}^{i}\right) \\
P_{k \mid k}^{i, \sigma_{i}} & =P_{k \mid k-1}^{i}-K_{k}^{i} S_{k}^{i}\left(K_{k}^{i}\right)^{\prime}
\end{aligned}
$$

For $\sigma_{i}=0, m_{k \mid k}^{i, \sigma_{i}}=m_{k \mid k-1}^{i}$ and $P_{k \mid k}^{i, \sigma_{i}}=P_{k \mid k-1}^{i}$.

Note that (27) includes a summation over $\Theta$, the set of all possible measurement associations $\theta$. Except for very simple scenarios with few targets and high signal to noise ratio, this is computationally infeasible. To mitigate computational complexity, data association is used. The JPDA algorithm was used in [7], however this solution is highly susceptible to track coalescence, and it also suffers from high computational complexity when there are many targets.

Instead, for each MOP, the auction algorithm [3] is used to compute the single most probable association event $\hat{\theta}^{\ell}$. Under this approximation the Bayes normalization constant $f\left(\mathbf{Z}_{k} \mid \mathbf{Z}^{k-1}\right)$ is zero for $\left|\mathbf{X}_{k}\right| \neq\left|\mathbf{X}_{k \mid k-1}^{\ell}\right|$, and for $\left|\mathbf{X}_{k}\right|=\left|\mathbf{X}_{k \mid k-1}^{\ell}\right|$ it becomes

$$
\int f\left(\mathbf{Z}_{k} \mid \mathbf{X}_{k}\right) \phi_{\mathbf{X}_{k}}\left(\mathbf{X}_{k \mid k-1}^{\ell}\right) \delta \mathbf{X}_{k}=\mathcal{L}_{k \mid k-1}^{\ell}\left(\hat{\theta}^{\ell}\right)
$$


We thus have a multi-object particle approximation of the posterior multi-object density

$$
\begin{aligned}
f\left(\mathbf{X}_{k} \mid \mathbf{Z}^{k}\right) & =\frac{\sum_{\ell=1}^{M} \mathcal{L}_{k \mid k-1}^{\ell}\left(\hat{\theta}^{\ell}\right) \phi \mathbf{X}_{k}\left(\mathbf{X}_{k \mid k}^{\ell, \hat{\theta}^{\ell}}\right)}{\sum_{\ell=1}^{M} \mathcal{L}_{k \mid k-1}^{\ell}\left(\hat{\theta}^{\ell}\right)} \\
& =\sum_{\ell=1}^{M} \mathcal{W}_{k \mid k}^{\ell} \phi \mathbf{X}_{k}\left(\mathbf{X}_{k \mid k}^{\ell, \hat{\theta}^{\ell}}\right)
\end{aligned}
$$

where

$$
\mathcal{W}_{k \mid k}^{\ell} \triangleq \frac{\mathcal{L}_{k \mid k-1}^{\ell}\left(\hat{\theta}^{\ell}\right)}{\sum_{\ell=1}^{M} \mathcal{L}_{k \mid k-1}^{\ell}\left(\hat{\theta}^{\ell}\right)}
$$

is the updated or posterior weight for the $l$ th MOP.

\subsubsection{Approximate posterior multi-Bernoulli density}

For each MOP within each gating group, it has a unique combination of the updated estimates with the posterior weights. A predicted MB component may be included in multiple MOPs, and in different MOPs, the same predicted MB component may be updated into different estimates, due to the data association. Therefore, there may be multiple updated estimates that correspond to the same predicted estimate.

The posterior multi-Bernoulli density with unimodal target estimates

$$
\left\{\left(w_{k \mid k}^{i}, m_{k \mid k}^{i}, P_{k \mid k}^{i}\right)\right\}_{i=1}^{N_{k \mid k-1}^{M B}}
$$

is obtained as follows,

$$
\begin{aligned}
w_{k \mid k}^{i} & =\sum_{\ell: i \in I_{\ell}} \mathcal{W}_{k \mid k}^{\ell} \\
m_{k \mid k}^{i} & =\frac{1}{w_{k \mid k}^{i}} \sum_{\ell: i \in I_{\ell}} \mathcal{W}_{k \mid k}^{\ell} m_{k \mid k}^{i, \hat{\sigma}_{i}^{\ell}} \\
M_{k \mid k}^{i, \hat{\sigma}_{i}^{\ell}} & =\left(m_{k \mid k}^{i, \hat{\sigma}_{i}^{\ell}}-m_{k \mid k}^{i}\right)\left(m_{k \mid k}^{i, \hat{\sigma}_{i}^{\ell}}-m_{k \mid k}^{i}\right)^{\prime} \\
P_{k \mid k}^{i} & =\frac{1}{w_{k \mid k}^{i}} \sum_{\ell: i \in I_{\ell}} \mathcal{W}_{k \mid k}^{\ell}\left(P_{k \mid k}^{i, \hat{\sigma}_{i}^{\ell}}+M_{k \mid k}^{i, \hat{\sigma}_{i}^{\ell}}\right)
\end{aligned}
$$

In other words, for each predicted MB component, an updated probability of existence is found by taking the sum of updated MOP weights for the MOPs that the estimate was included in. A single updated Gaussian density is found by merging the updated Gaussian densities from the MOPs that the estimate was included in. Note that this merging minimizes the Kullback-Leibler divergence between the Gaussian mixture and the single Gaussian (see [25]).

\subsection{Pruning, confirmation and extraction}

To reduce the complexity after the update, multi-Bernoulli components with a probability of existence $w_{k \mid k}^{i}$ lower than a threshold $\tau_{\mathrm{P}}$ are pruned (i.e. removed). A target estimate is considered confirmed if the probability of existence is larger than a threshold $\tau_{\mathrm{C}}$ for at least one time step. An estimate of the target set is taken as the set of estimates that have either been confirmed or have probabilities of existence larger than a threshold $\tau_{\mathrm{E}}$. 


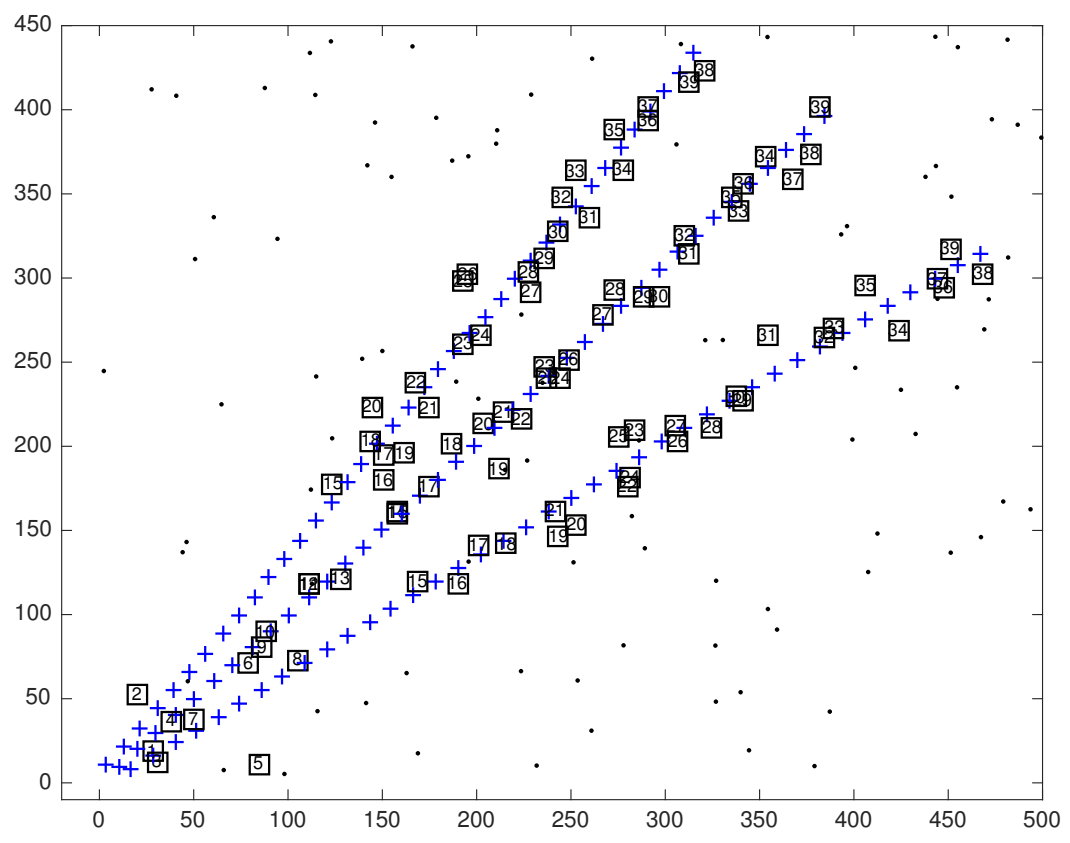

(a) Merged measurements: + target true position; $\square$ target originated measurements with corresponding time indices; · clutter measurements

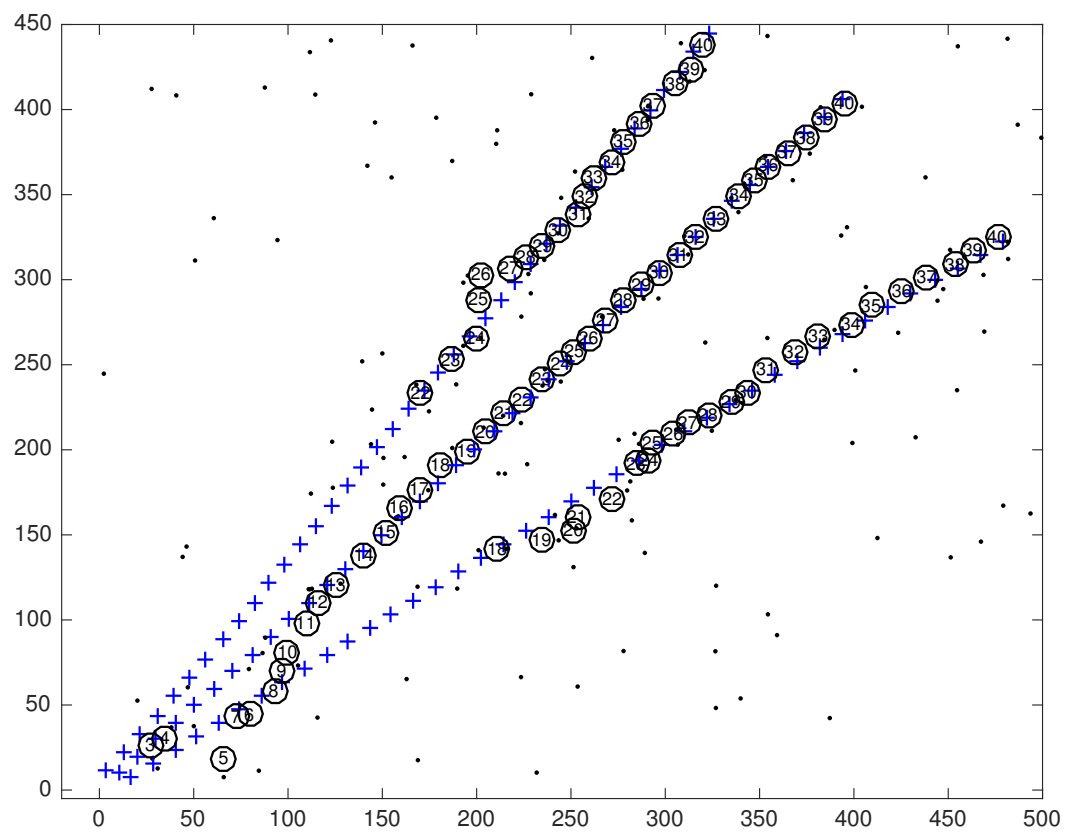

(b) Multi-Bernoulli filtering: + target true position; $\bigcirc$ estimated track with corresponding time indices; · the measurement

Figure 1. One run for $\lambda_{c}=10$ over 40 frames. 


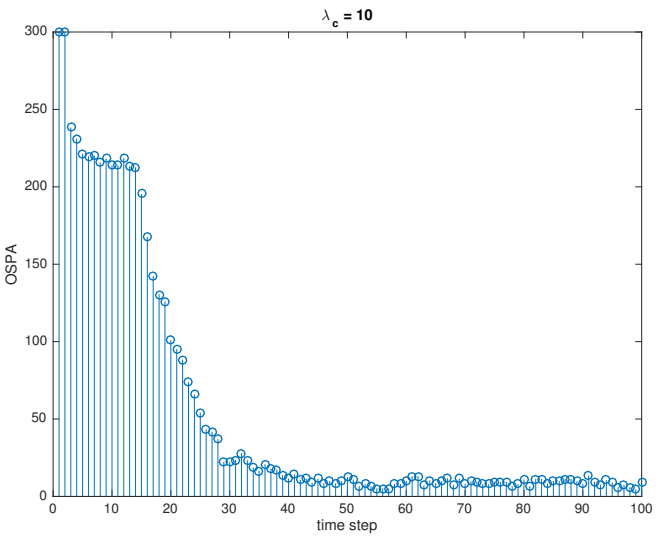

(a) OSPA

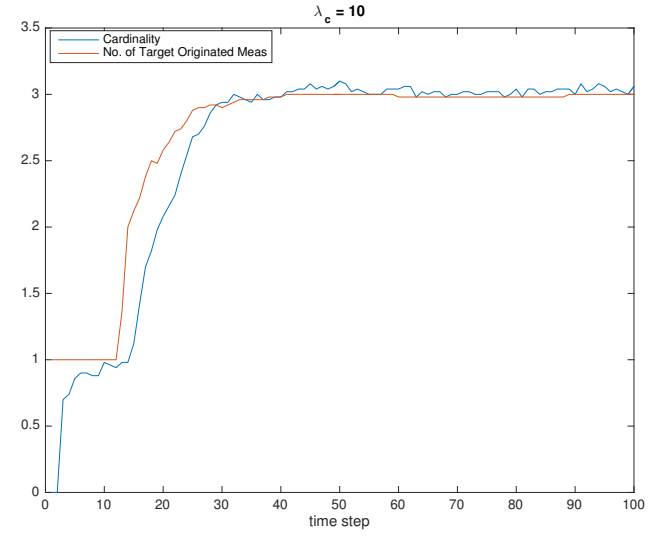

(b) Cardinality

Figure 2. 50 Monte Carlo runs with clutter intensity 10.

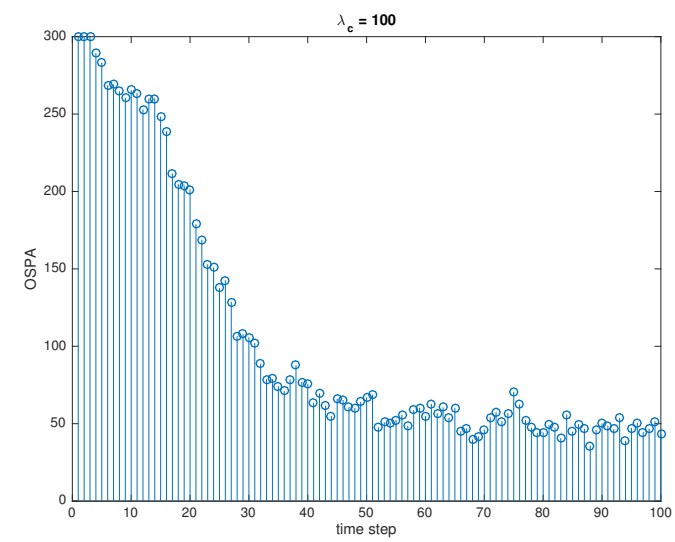

(a) OSPA

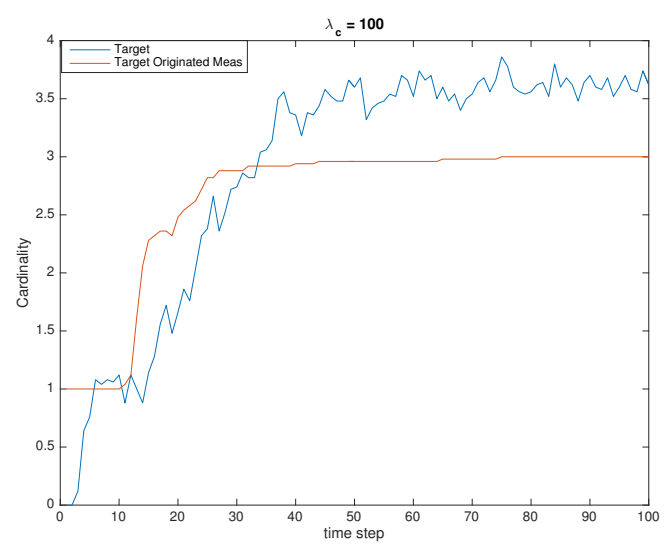

(b) Cardinality

Figure 3. 50 Monte Carlo runs with clutter intensity 100.

\section{SIMULATION RESULTS}

Simulation results were conducted for the merged-measurements scenario described in Section 2 using MOP-MB filter. Three targets were to be tracked in an optical sensor's focal plane, i.e., in 2-D Cartesian coordinates. A WNA model was considered with acceleration noise standard deviation $\sigma_{v}=0.1 \mathrm{~m} / \mathrm{s}^{2}$. The distances of the initial positions of the three targets were very close and the three targets had the same initial speed $10 \sqrt{2} \mathrm{~m} / \mathrm{s}$ with slightly different directions of the velocity vector. The initial states of the three targets were respectively: $\mathbf{x}_{0}^{1}=[0,0,10,10]^{\prime}, \mathbf{x}_{0}^{2}=\left[5,0, \sqrt{200-8^{2}}, 8\right]^{\prime}, \mathbf{x}_{0}^{3}=\left[-5,0, \sqrt{200-11^{2}}, 11\right]^{\prime}$. At the first few time steps, the three targets were so close that they were not able to be resolved by the sensor, resulting in merged measurements. The resolution model is as discussed in Section 2.2. At each time step, the measurement noise standard deviation for a resolved target was $\sigma_{r}=10 \mathrm{~m}$. The number of clutter measurements at each time step was Poisson distributed with mean $\lambda_{c}$ and the clutter measurements were uniformly distributed in the surveillance area $[0,1000] m \times[0,1000] m$. The sampling time was $T=1 s$, and the probability of survival for each target was $p_{\mathrm{S}}=0.99$. The detection probability for each target was $p_{D}=0.999$. For data association, the gating probability $P_{\mathrm{G}}=0.999$ was used. For pruning, confirmation and extraction the following thresholds were used: $\tau_{\mathrm{P}}=10^{-4}$, $\tau_{\mathrm{C}}=0.6$, and $\tau_{\mathrm{E}}=0.2$. The initial existence probability $w_{b}$ was set to be 0.1 and the initial covariance matrix 
was $^{\ddagger}$

$$
P_{b}=\left[\begin{array}{llll}
3 \sigma_{r}^{2} & 0 & 0 & 0 \\
0 & 3 \sigma_{r}^{2} & 0 & 0 \\
0 & 0 & 100 & 0 \\
0 & 0 & 0 & 100
\end{array}\right]
$$

Fig. 1(a) shows the positions of the three targets and the corresponding target originated measurements at each time step for one particular run for clutter intensity $\lambda_{c}=10$. As shown in the figure, for a typical run, there were no resolved measurements for the three targets up to time step 14. After that, the targets separated and all the target-originated measurements became resolved. Fig. 1(b) shows the extracted tracks by the MB filter on top of the true positions and measurements in the same run. The first track was confirmed at time step 3 and it was an unresolved track. The second track was extracted at time step 18. At time step 22, all three tracks had been extracted.

Next, 50 Monte Carlo runs were conducted to evaluate the average performance for different clutter intensities: the target cardinality estimates and the optimal subpattern assignment (OSPA) metric $[20]^{\S}$. Cardinality estimates were taken as the number of extracted targets. As for OSPA, it was used to measure the distance between any two sets $X=\left\{x_{1}, \ldots, x_{m}\right\}$ and $Y=\left\{y_{1}, \ldots, y_{n}\right\}$, which is defined in the following

$$
\bar{d}_{p}^{(c)}(x, y) \triangleq\left(\frac{1}{n}\left(\min _{\pi \in \Pi_{n}} \sum_{i=1}^{m} d^{(c)}\left(x_{i}, y_{\pi(i)}\right)^{p}+c^{p}(n-m)\right)\right)^{1 / p}
$$

where $d^{c}(x, y) \triangleq \min (c, d(x, y))$ is the distance between $x, y$ cut off at $c$ and $\Pi_{n}$ is the set of permutations on $\{1,2, \ldots, n\}$. For this paper, it was implemented using the Euclidean norm with cut-off parameter $c=300$ and $p=1$.

As shown in Figs. 2 and 3, the MB filter had about the same convergence rate for the number of tracks at different clutter intensities. But when the clutter rate was high, it was more susceptible to false alarms, which led to short-lived false tracks.

\section{CONCLUSION}

This paper considered a MTT problem with merged measurements in an optical sensor's focal plane. A preliminary model was used for the merged (unresolved) measurements. A recently proposed MB filter was applied to this problem. Simulation results demonstrated that the MB filter was capable of keeping track of the initially unresolved tracks a few time steps later after they separate. In the future, we will build a physics-based model for the unresolved measurements. Also, we will explore more complicated dynamic models and motions in 3D, such as thrusting. Finally, we plan to develop a procedure to map the $2 \mathrm{D}$ trajectories from the focal plane into the 3D space using the approach of [26].

\section{REFERENCES}

[1] Y. Bar-Shalom, X. Rong-Li, and T. Kirubarajan, Estimation with Applications to Tracking and Navigation. New York, NY, USA: John Wiley \& Sons, 2001.

[2] Y. Bar-Shalom, P. K. Willett, and X. Tian, Tracking and Data Fusion: A Handbook of Algorithms. YBS Publishing, 2011.

[3] D. Bertsekas, "The auction algorithm: A distributed relaxation method for the assignment problem," Annals of Operations Research, vol. 14, no. 1, pp. 105-123, 1988.

[4] S. Blackman and R. Popoli, Design and Analysis of Modern Tracking Systems. Norwood, MA, USA: Artech House, 1999.

\footnotetext{
${ }^{\ddagger}$ It is known that unresolved measurements have larger errors than resolved ones [9]. A future physics-based study in this direction is planned.

$\S$ This approach assigns each estimated track to the nearest true target track, i.e., it has no "labels" for the tracks.
} 
[5] R. Fitzgerald, "Development of practical PDA logic for multitarget tracking by microprocessor," in Multitarget-Multisensor Tracking: Advanced Applications, Y. Bar-Shalom, Ed. Artech House, 1990, pp. $1-23$.

[6] D. Fränken, M. Schmidt, and M. Ulmke, "'Spooky Action at a Distance' in the Cardinalized Probability Hypothesis Density Filter," IEEE Transactions on Aerospace and Electronic Systems, vol. 45, no. 4, pp. 1657-1664, Oct. 2009.

[7] K. Granström, P. Willett, and Y. Bar-Shalom, "PHD filter with approximate multiobject density measurement update," in Proceedings of the International Conference on Information Fusion, Washington, DC, USA, Jul. 2015, pp. 1802-1809.

[8] K. Granström, P. Willett, and Y. Bar-Shalom, "Approximate multi-hypothesis multi-bernoulli multi-object filtering made multi-easy," IEEE Transactions on Signal Processing, vol. 64, no. 7, pp. 1784-1797, April 2016.

[9] M. Kovacich, T. Casaletto, B. Lutjens, and D. McIntyre, "An application of mht to group to object tracking," Proceedings of SPIE Conference on Signal and Data Processing of Small Targets, vol. 1481, Aug. 1991.

[10] Q. Le and L. Kaplan, "Probability Hypothesis Density-Based Multitarget Tracking for Proximity Sensor Networks," IEEE Transactions on Aerospace and Electronic Systems, vol. 49, no. 3, pp. 1476-1496, Jul. 2013.

[11] R. Mahler, Statistical Multisource-Multitarget Information Fusion. Norwood, MA, USA: Artech House, 2007.

[12] —, Advances in Multisource-Multitarget Information Fusion. Norwood, MA, USA: Artech House, 2014.

[13] — - "Multitarget Bayes filtering via first-order multi target moments," IEEE Transactions on Aerospace and Electronic Systems, vol. 39, no. 4, pp. 1152-1178, Oct. 2003.

[14] — "PHD filters of higher order in target number," IEEE Transactions on Aerospace and Electronic Systems, vol. 43, no. 4, pp. 1523-1543, Oct. 2007.

[15] K. Murty, "An algorithm for ranking all the assignments in order of increasing cost," Operations Research, vol. 16, no. 3, pp. 682-687, 1968.

[16] K. Panta, D. Clark, and B.-N. Vo, "Data association and track management for the Gaussian mixture probability hypothesis density filter," IEEE Transactions on Aerospace and Electronic Systems, vol. 45, no. 3, pp. 1003-1016, Jul. 2009.

[17] V. C. Ravindran, L. Svensson, L. Hammarstrand, and M. Morelande, "A cardinality preserving multitarget multi-Bernoulli RFS tracker," in Proceedings of the International Conference on Information Fusion, Singapore, Jul. 2012, pp. 832-839.

[18] S. Reuter, B.-T. Vo, B.-N. Vo, and K. Dietmayer, "The Labeled Multi-Bernoulli Filter," IEEE Transactions on Signal Processing, vol. 62, no. 12, pp. 3246-3260, Jul. 2014.

[19] B. Ristic, D. Clark, B.-N. Vo, and B.-T. Vo, "Adaptive target birth intensity for PHD and CPHD filters," IEEE Transactions on Aerospace and Electronic Systems, vol. 48, no. 2, pp. 1656-1668, Apr. 2012.

[20] D. Schuhmacher, B.-T. Vo, and B.-N. Vo, "A consistent metric for performance evaluation of multi-object filters," IEEE Transactions on Signal Processing, vol. 56, no. 8, pp. 3447-3457, Aug. 2008.

[21] B.-T. Vo and B.-N. Vo, "Labeled random finite sets and multi-object conjugate priors," IEEE Transactions on Signal Processing, vol. 61, no. 13, pp. 3460-3475, Apr. 2013.

[22] B.-T. Vo, B.-N. Vo, and A. Cantoni, "The cardinality balanced multi-target multi-bernoulli filter and its implementations," IEEE Transactions on Signal Processing, vol. 57, no. 2, pp. 409-423, Feb. 2009.

[23] B.-T. Vo, B.-N. Vo, R. Hoseinnezhad, and R. Mahler, "Robust multi-bernoulli filtering," IEEE Journal of Selected Topics in Signal Processing, Special Issue on Multi-target Tracking, vol. 7, no. 3, pp. 399-409, Jun. 2013.

[24] B.-T. Vo, B.-N. Vo, and D. Phung, "Labeled random finite sets and the Bayes multi-target tracking filter," IEEE Transactions on Signal Processing, vol. 62, no. 24, pp. 6554-6567, Dec. 2014.

[25] J. Williams, "An efficient, variational approximation of the best fitting multi-Bernoulli filter," IEEE Transactions on Signal Processing, vol. 63, no. 1, pp. 258-273, Jan. 2015.

[26] T. Yuan, Y. Bar-Shalom, P. Willett, R. Ben-Dov, and S. Pollak, "Estimation of thrusting trajectories in 3d from a single fixed passive sensor," IEEE Transactions on Aerospace and Electronic Systems, vol. 50, no. 3, pp. 2096-2108, Jul. 2014. 